\title{
Control of recA dependent activities in Escherichia coli: a possible role for the recF product
}

\author{
By ALLYN THOMAS AND ROBERT G. LLOYD* \\ Department of Genetics, University of Nottingham, Nottingham NG7 2RD, U.K.
}

(Received 29 April 1982; revised 2 August 1982)

\begin{abstract}
DNA repair and genetic recombination have been studied in recF 143 mutants of Escherichia coli in which expression of inducible, SOS repair activities was altered by additional mutations either in lexA or recA. recF143 and lexA3 appeared to act additively to increase sensitivity to UV irradiation and to reduce recombination proficiency. The recF defect was suppressed in strains carrying the tif-1 allele of $r e c A$ but not in strains carrying mutations that increased synthesis of rec $A^{+}$protein or which directly inactivated lexA repressor. These and other data are interpreted to suggest that the $r e c F$ defect is related to a reduced activity of rec $A$ protein. The implications of these findings are discussed in relation to the control of SOS activities and cell division during normal growth.
\end{abstract}

\section{INTRODUCTION}

Cell division in Escherichia coli is arrested following damage to DNA in conjunction with an increase in various activities that promote DNA repair (Witkin, 1976). The regulatory basis for this coordinate 'SOS' response is now understood: an inhibitor of division produced by the sfiA gene is regulated in parallel with the products of several DNA repair and other genes through a repressor specified by lexA. Damage to DNA activates a protease function of recA protein, which cleaves this repressor and causes the derepression of all lexA regulated genes (Huisman \& D’Ari, 1981; Kenyon \& Walker, 1980; Little \& Harper, 1979; Phizicky \& Roberts, 1981; Little et al., 1980).

Although lexA and recA specify the main regulatory elements that control the SOS response, normal expression of several SOS activities is reduced by mutation of the recF gene. The recF gene was first identified during the analysis of recombination in $r e c B / C s b c B$ mutants (Horii \& Clark, 1973). Subsequent studies revealed many similarities between rec $F$ mutants and those lex $A$ mutants that are unable to induce the SOS response. They are sensitive to UV light and, after irradiation, show reduced amplification of $\operatorname{rec} A$ protein and less cell filamentation than is usual. UV induction of $\lambda$ prophage is reduced, as is post-replication repair of DNA damage and Weigle-reactivation of irradiated phage (Rothman \& Clark, 1977; Armengod \& Blanco, 1978; Rothman et al., 1979; Kato \& Shinoura, 1977). lexA and recF mutations also block recombination in $r e c B / C s b c B$ mutants (Clark, 1980) and reduce its efficiency in $r e c B / C^{+} s b c B^{+}$ strains (this work).

To gain further insight into the nature of the $r e c F$ defect and its effect on SOS expression we have studied DNA repair and genetic recombination in recF143 mutants in which expression of SOS genes was altered through mutation of $\operatorname{lexA}$ or $\operatorname{rec} A$. The results obtained indicate that the $r e c F$ defect may be related to reduced activity of $\operatorname{rec} A$ protein and we suggest that the $r e c F$ product normally acts to control lexA regulated processes in the absence of any damage to DNA.

\section{METHODS}

Bacterial strains. The Escherichia coli $\mathrm{K}_{12} \mathrm{~F}^{-}$strains are listed in Table 1. All are closely related to AB1157 (Bachmann, 1972) (F-thi-1 his-4 proA2 argE3 thr-1 leuB6 ara-14 galK2 lacY1 mtl-1 xyl-5 tsx-33 rpsL31 supE44). 
JC9239, carrying recF143, was obtained from A. J. Clark. Derivatives of AB1157 and JC9239 carrying various alleles of lexA or recA were constructed by $\mathrm{P} 1$ transductions from the appropriate donors into strains previously made malE : :Tn10 or $s r l:: \operatorname{Tn} 10$, with selection for $\mathrm{Mal}^{+}$and $\mathrm{Srl}^{+}$, respectively. $s f B^{-}$strains were constructed by mating with $\mathrm{Hfr}$ (Hayes) $s f B^{-}$(J. George strains GC895) with selection for $\mathrm{Leu}^{+}$(rpsL) transconjugants. The genotype of all strains was confirmed by the appropriate P1 transductional backcrosses. The multicopy plasmids pJL21 (lex $\left.A^{+}\right)$and pJL26 (lexA3) are derivatives of pBR322 (Mount et al., 1980) and were introduced into the appropriate recipients by transformation and selection for tetracycline resistant clones.

Media. LB broth and agar, used for the routine culture of bacterial strains, and 56/2 minimal salts agar for the selection of recombinants and transconjugants from crosses, have been referred to previously (Lloyd \& Low, 1976).

Conjugal recombination. The procedure for measuring the efficiency of recombination in $\mathrm{Hfr}$ crosses has been described (Lloyd \& Low, 1976). $\mathrm{F}^{-}$recipients were mated with Hfr strain KL226 (Hfr Cavalli) for $60 \mathrm{~min}$ at $30^{\circ} \mathrm{C}$ after which samples were plated on defined agar medium selective for $\operatorname{Pro}^{+}(r p s L)$ recombinants. Samples of the same $\mathrm{F}^{-}$cultures were also mated with the F-lac ${ }^{+}$donor strain NH4104 (Lloyd \& Low, 1976) for $30 \mathrm{~min}$ at $30^{\circ} \mathrm{C}$ and selection made for $\mathrm{Lac}^{+}(r p s L) \mathrm{F}$-prime exconjugants. Colonies were scored after $48 \mathrm{~h}$ incubation. The yield of recombinants from $\mathrm{Hfr}$ crosses expressed as a fraction of that obtained with AB1157 was used as a measure of recombination deficiency. The yield of $\mathrm{Lac}^{+}$exconjugants in the F-prime crosses was used to correct for any reduction in the mating efficiency of the strains relative to $\mathrm{AB} 1157$.

$U V$ irradiation. Strains were grown to about $2 \times 10^{8}$ cells $\mathrm{ml}^{-1}$ in $\mathrm{LB}$ broth medium and irradiated on the surface of LB agar plates as described previously (Lloyd \& Low, 1976; Lloyd \& Barbour, 1974). Strains harbouring lexA plasmids were grown in the presence of tetracycline $\left(10 \mu \mathrm{g} \mathrm{ml}^{-1}\right)$ to prevent growth of plasmid-free segregants. Colonies of survivors were scored after $24-36 \mathrm{~h}$ at $30^{\circ} \mathrm{C}$.

Nitrofurantoin sensitivity. The procedure used was modified from that described by Ream et al. (1980). Strains were grown in LB broth medium to about $2 \times 10^{8}$ cells ml-1 and samples of various dilutions were plated on LB agar containing various concentrations of nitrofurantoin (Sigma). Colonies of survivors were scored after $48 \mathrm{~h}$ incubation.

\section{RESULTS}

\section{Additivity of lexA3 and recF143 defects}

A series of closely related strains carrying various combinations of $l e x A$ and $r e c F$ alleles were examined for their sensitivity to UV irradiation and for their recombination proficiency in crosses with an Hfr donor. The lexA3 recF 143 double mutant proved more sensitive to UV light and less proficient at recombination than strains carrying either of these mutations alone (Fig. $1 a$, Table 1 a). Mount $e t$ al. (1980) showed that although the lexA gene is autoregulated the level of repressor can be increased, and SOS expression reduced, by introducing multicopy lexA plasmids into the cell. The data presented in Fig. $1(b, c)$ and Table $1(b)$ demonstrate that the additivity of $l e x A$ and $r e c F$ defects is also observed in strains carrying multicopy lex $A$ plasmids. A plasmid carrying the mutant lexA 3 allele had a more extreme effect on UV sensitivity and recombination proficiency than a lex $A^{+}$plasmid, but in both cases the effect was enhanced by a $r e c F$ mutation. The $\operatorname{lexA}$ recF double mutant carrying the lexA3 plasmid proved extremely sensitive to UV light, and recombination was reduced to $3-4 \%$ of that in a wild-type (plasmid free) strain.

\section{Increased synthesis of rec $A$ protein does not alleviate the recF defect}

Previous studies (Thomas \& Lloyd, 1980) showed that strains carrying alc-24, a rec $A$ regulatory site mutation, synthesize high levels of $r e c A$ protein even in the presence of lexA3 which normally prevents derepression of $\operatorname{rec} A$. The UV sensitivity and recombination deficiency of $\operatorname{lex} A$ and $\operatorname{lex} A$ rec $F$ mutants, particularly those carrying lex $A$ plasmids, was alleviated to some extent by alc-24 (Fig. $1 d$, Table $1 \mathrm{c}$ ). However, the alc-24lexA $A^{+}\left(\right.$recF $\left.F^{+}\right)$strain used for comparison proved more proficient at recombination and more resistant to UV light than any of these strains. alc-24 appeared to have no effect on a rec $F$ mutant except where the strain was also $l e x A^{-}$. The alc-24 recF lexA $A^{+}$strain proved as sensitive to $\mathrm{UV}$ light and as deficient for recombination as the corresponding alc ${ }^{+}$strains (Fig. 1e, Table 1).

\section{tif expression alleviates the DNA repair defect in recF143 strains}

The observations described in the previous section demonstrate that both lex $A$ and $\operatorname{rec} F$ mutations prevent normal recombination and DNA repair even when the amount of $\operatorname{rec} A$ 


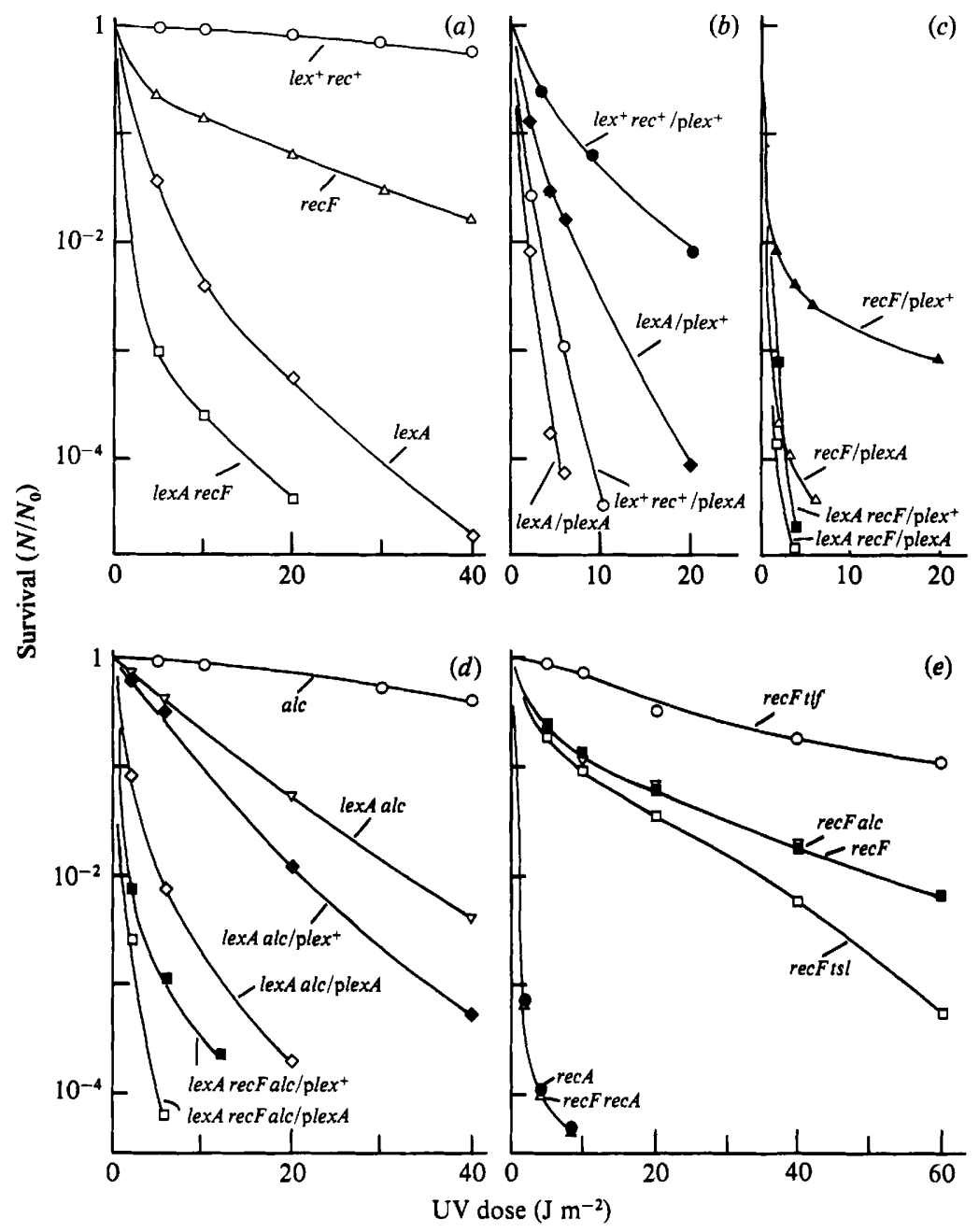

Fig. 1. UV survival of recF mutants and related strains. Cultures were grown in $\mathrm{LB}$ broth at $30^{\circ} \mathrm{C}$ and irradiated on the surface of LB agar plates as referred to in Methods. The UV fluence was $1 \mathbf{J}$ per $\mathbf{m}^{2}$. (a) O, AB1157; $\triangle$, JC9239; $\diamond, \mathrm{RL} 201 ; \square, \mathrm{RL} 202$. (b) O, AB1157/pJL21; O, AB1157/pJL26; RL201/pJL21; $\diamond$ RL201/pJL26. (c) $\Delta$, JC9239/pJL21; $\triangle$, JC9239/pJL26; $\boldsymbol{Q}, \mathrm{RL202/pJL21;} \square$,

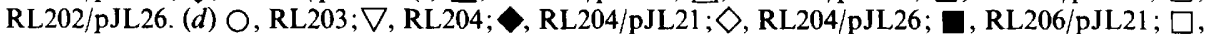
RL206/pJL26. (e) O, RL207; $\nabla$, RL205;

protein is not a limiting factor. The lexA defect can be attributed to reduced expression of one or more of the other activities regulated by lex $A$ repressor. To investigate whether or not the same could apply to $r e c F$ strains, $r e c F$ tif and $\operatorname{rec} F$ tsl $(\operatorname{lex} A)$ mutants were constructed and examined for their ability to repair UV damage. The tif-I mutation is an allele of recA that increases the protease activity of $r e c A$ protein (Phizicky \& Roberts, 1981) while $t s l-1$ is an intragenic suppressor of lexA3 which directly inactivates the altered repressor (Mount et al., 1973). Both mutations cause increased expression of lexA regulated genes, particularly at high temperature $\left(42{ }^{\circ} \mathrm{C}\right)$. Figure $1(e)$ shows that even at $30^{\circ} \mathrm{C}$ the UV sensitivity of a recF mutant was suppressed by tif but not by $t s l$. The survival of $t s l$ rec $F^{+}$and $t i f r e c F^{+}$strains did not differ significantly from that of wild-type strains under the conditions used (data not shown). To determine whether or not $t s l$ suppresses the rec $F$ DNA repair defect at $42{ }^{\circ} \mathrm{C}$ we made use of the fact that a rec $F$ mutant is more sensitive to nitrofurantoin than is a recF $F^{+}$strain (Ream et al., 1980) (for some unknown reason, a recF mutant was slightly more resistant to UV light at $42^{\circ} \mathrm{C}$ than at $30^{\circ} \mathrm{C}$, data not 


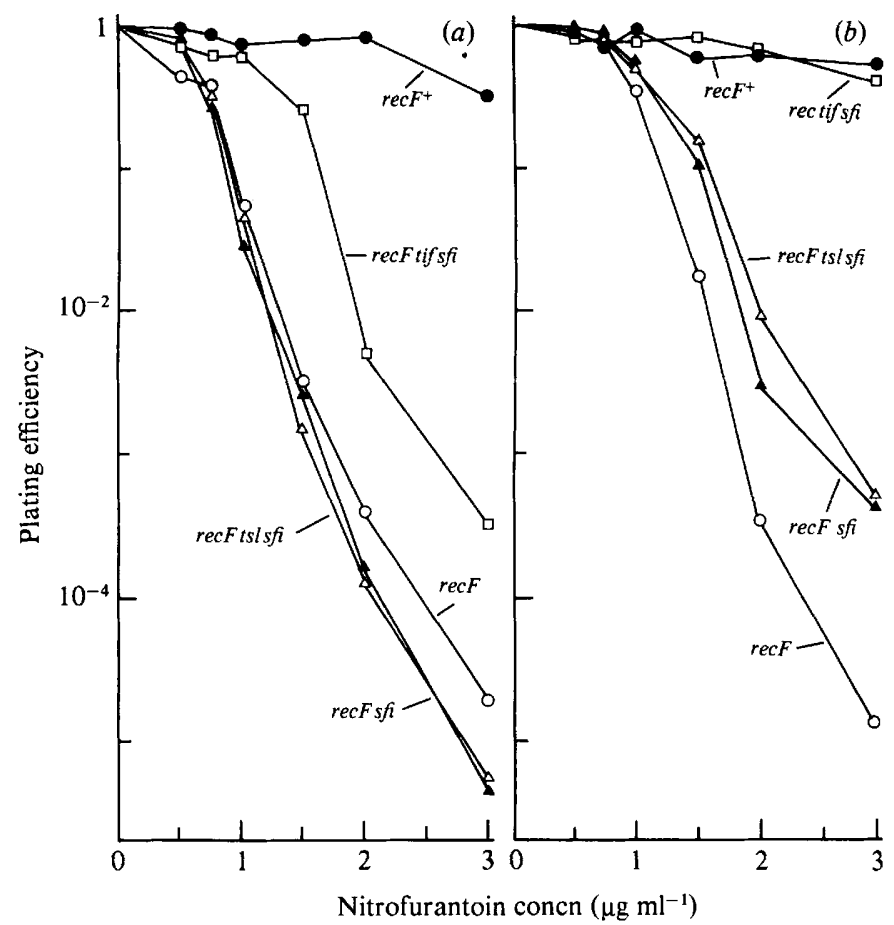

Fig. 2. Nitrofurantoin sensitivity of $r e c F$, recF tif and $r e c F t s l$ strains. Cells were grown and spread on agar plates containing various concentrations of nitrofurantoin. Incubation was at $30^{\circ} \mathrm{C}(a)$ or $42{ }^{\circ} \mathrm{C}(b)$. The strains used were: $\mathrm{O}, \mathrm{AB} 1157 ; \mathrm{O}$, JC9239; $\mathbf{A}$, RL210; $\square, \mathrm{RL} 211 ; \triangle, \mathrm{RL212}$.

Table 1. Strains used and recombination efficiency

Strain

(a) $\mathrm{AB} 1157$

JC9239

RL201

RL.202

(b) AB1157/pJL21

AB1 157/pJL26

JC9239/pJL21

JC9239/pJL26

RL201/pJL21

RL201/pJL26

RL202/pJL21

RL202/pJL26

(c) RL203

RL204

RL204/pJL21

RL204/pJL26

RL205

RL206

RL206/pJL21

RL206/pJL26

(d) RL207

RL208

RL209

AB2463

RL210

RL211

RL212
Relevant properties

Wild-type $\left(\operatorname{lexA^{+}} r e c F^{+} \operatorname{rec} A^{+}\right)$

recF 143

lexA3

lexA3 recF 143

$\operatorname{lexA^{+}/\text {plexA}}{ }^{+}$

lexA $A^{+} / \mathrm{plexA3}$

recF143/plexA ${ }^{+}$

recF143/plexA3

lexA3/plexA ${ }^{+}$

lexA3/plexA3

lexA3 recF 143/plexA $A^{+}$

lexA3 recF $143 / \mathrm{plexA3}$

alc-24

lexA3 alc- 24

lexA3 alc-24/plexA $A^{+}$

lexA3 alc-24/plexA3

recF 143 alc- 24

lexA3 recF143 alc- 24

lexA3 recF143 alc-24/plexA ${ }^{+}$

lexA3 recF 143 alc-24/plexA3

recF143 tif-I

recF143 (lexA3) tsl-1

recF 143 rec $A 13$

recA13

recF143 sfiB

recFI43 tif-1 sfiB

recF 143 (lexA3) tsl-I sfiB
Recombination deficiency

$$
\begin{aligned}
& 1 \\
& 0.51 \\
& 0.42 \\
& 0.12 \\
& 0.73 \\
& 0.17 \\
& 0.16 \\
& 0.037 \\
& 0.34 \\
& 0.15 \\
& 0.09 \\
& 0.03 \\
& 1.00 \\
& 0.55 \\
& 0.63 \\
& 0.48 \\
& 0.49 \\
& \text { ND } \\
& 0.35 \\
& 0.24 \\
& 0.69 \\
& 0.76 \\
& \text { ND } \\
& \text { ND } \\
& \text { ND } \\
& \text { ND } \\
& \text { ND }
\end{aligned}
$$

ND, Not determined. 
shown). Figure 2 shows that the recF $t s l(s f i B)$ strain remained sensitive to nitrofurantoin at both $30^{\circ} \mathrm{C}$ and $42^{\circ} \mathrm{C}$, whereas the recF tif (sfiB) strain was resistant at $42^{\circ} \mathrm{C}$ and showed increased tolerance to the drug at $30^{\circ} \mathrm{C}$. The presence of the $s f B$ mutation, which is required for division of $t i f$ and $t s l$ strains at high temperature (George $e t$ al., 1975), accounts for the slightly increased nitrofurantoin resistance of the recF tsl strain at $42^{\circ} \mathrm{C}$ (Fig. $2 b$ ).

These results demonstrate that the DNA repair defect of a $r e c F$ mutant is suppressible by $t i f$ but not by $t s l$. This contrasts with the situation found in a lexA mutant where the repair defect is suppressed by $t s l$ but not by tif. A further difference between the lex $A$ and $r e c F$ defects is that the former considerably enhances the UV sensitivity of a $\operatorname{rec} A$ strain (data not shown), whereas the latter does not (Fig. 1e).

\section{DISCUSSION}

Since transcription of SOS genes appears to be controlled by lexA repressor it is difficult to imagine how a $r e c F$ mutation could modify expression of SOS activities other than by affecting the level of repressor. The results presented in this paper are consistent with this view. The phenotype of a $r e c F$ mutant is less severe than that of a lexA mutant, but is somewhat similar to that of a rec $F^{+}$strain carrying a multicopy lex $A^{+}$plasmid. This, together with the additive effect of lex $A$ and $\operatorname{rec} F$ mutations, suggests that the $\operatorname{rec} F$ defect may be due to increased expression of the lex $A^{+}$repressor. We argue, therefore, that the function of the $r e c F^{+}$product is to reduce the effective level of repressor in the cell. Since a $r e c F$ mutation is suppressed by $t i f$ and not by $t s l$ we further argue that $r e c F^{+}$product achieves this effect by increasing $r e c A$ protease activity. This is supported by the fact that a $r e c F$ mutation does not enhance the UV sensitivity of a recA strain (Fig. $1 e$ ). The SOS genes would still be inducible in $r e c F$ mutants, though the response would be retarded due to reduced basal levels of $\operatorname{rec} A$ protease activity. This would account for the normal UV mutability of recF mutants (Clark, 1980) and for the fact that the UV sensitivity of recF mutants is particularly noticeable at low UV doses (Fig. $1 a$ ). Since rec $A$ protease cannot cleave the mutant lexA3 repressor (Little et al., 1980), the additivity of lexA and recF defects would suggest that activated $r e c A$ protein can enhance repair activities in the absence of increased expression of other lex $A$ regulated genes. Alternatively, the rec $F$ product may have an additional role in DNA repair which is independent of $\operatorname{rec} A$ protein, but this is not consistent with the properties of $r e c F$ recA double mutants.

To cleave repressors in vitro, recA protein must be activated by polynucleotide or single-strand fragments of DNA (Phizicky \& Roberts, 1981). The recF product could be responsible for such an activator molecule being produced at low levels during normal DNA replication, or it may be a protein which interacts directly with $\operatorname{rec} A$ protein to promote its activity. Alternatively, the recF $F^{+}$product could be an RNA molecule that acts as a specific effector of $\operatorname{rec} A$ protein in the absence of DNA damage. McEntee \& Weinstock (1981) have shown that tif- 1 protein is activable by polyribonucleotides, and it may be that $r e c A^{+}$protein is similarly activated in vivo by a specific polyribonucleotide sequence.

This interpretation of $r e c F$ function implies that some $\operatorname{rec} A$ protease would be produced during normal growth and provide a means for varying the expression of SOS regulated genes in the absence of any overt damage to DNA. What purpose would be served by the additional level of control? Since lexA and $\operatorname{rec} A$ products regulate $s f A$, and both $l e x A$ and $\operatorname{rec} A$ mutants divide prematurely to produce smaller cells, many of which contain no DNA (Howe \& Mount, 1978), one possibility is that the $r e c F^{+}$product has a role in controlling the normal cycle of cell division. The location of recF near the origin of DNA replication between dnaA and gyrB (Ream et al., 1980) would be consistent with such a role (Zaritsky \& Pritchard, 1973). A link between initiation of DNA replication and recA protease activity is suggested by the observation that mutations which inhibit initiation at high temperature (dnaA46 dnaB252) prevent tif expression (D'Ari et al., 1979).

A further feature of this model for $r e c F$ function is that it provides a simple explanation for the role of the recF product in genetic recombination and the different requirements of the so-called $\mathrm{RecB} / \mathrm{C}$ and RecF pathways (Clark, 1980). In the absence of the $r e c B / C$ product, exonuclease $\mathrm{V}$, the $r e c F$ product could promote the DNA unwinding activity of $r e c A$ protein to provide the 
single-stranded regions necessary for initiating recombination events (Cunningham et al., 1979; Radding, 1981).

The authors would like to thank the Science Research Council for their support, and J. W. Little and A. J. Clark for the gift of E. coli strains.

\section{REFERENCES}

ARMENGOD, M. E. \& BLANCO, M. (1978). Influence of the recF mutation of Escherichia coli $\mathrm{K} 12$ on prophage $\lambda$ induction. Mutation Research 52, 37-47.

BachmanN, B. J. (1972). Pedigree of some mutant strains of Escherichia coli K-12. Bacteriological Reviews 36, 525-557.

Clark, A. J. (1980). A view of the $\operatorname{RecB} / \mathrm{C}$ and $\mathrm{RecF}$ pathways of $E$. coli recombination. In Mechanistic Studies of DNA replication and Genetic Recombination (ICN-UCLA Symposium XIX), pp. 891-899. Edited by B. Alberts.

Cunningham, R. P., Takehiko, S., DasGupta, C. \& RADDING, C. M. (1979). Single strands induce recA protein to unwind duplex DNA for homologous pairing. Nature, London 28, 191-195.

D'Ari, R., George, J. \& HuIsman, O. (1979). Suppression of $t i f$-mediated induction of SOS functions in Escherichia coli by an altered $\operatorname{dnaB}$ protein. Journal of Bacteriology 140, 381-387.

George, J., Castellazzi, M. \& Buttin, G. (1975). Prophage induction and cell division in E. coli. III. Mutations sfiA and $s f B$ restore division in tif and lon strains and permit the expression of mutator properties of tif. Molecular and General Genetics 140, 309-332.

HoRII, Z. \& ClARK, A. J. (1973). Genetic analysis of the RecF pathway to genetic recombination in Escherichia coli $\mathrm{K} 12$ : isolation and characterization of mutants. Journal of Molecular Biology 80, 327-344.

Howe, W. E. \& Mount, D. W. (1978). Analysis of cell division in single clones of the Escherichia coli K-12 lexA mutant. Journal of Bacteriology 133, 1278-1281.

HUISMAN, O. \& D'ARI, R. (1981). An inducible DNA replication-cell division coupling mechanism in $E$. coli. Nature, London 290, 797-799.

KATO, T. \& SHINOURA, Y. (1977). Isolation and characterization of mutants of Escherichia coli deficient in induction of mutation by ultraviolet light. Molecular and General Genetics 156, 121-131.

KenYon, C. J. \& Walker, G. C. (1980). DNAdamaging agents stimulate gene expression at specific loci in Escherichia coli. Proceedings of the National Academy of Sciences of the United States of America 77, 2819-2833.

LITTLE, J. W. \& HARPER, J. E. (1979). Identification of the lexA gene product of Escherichia coli K-12. Proceedings of the National Academy of Sciences of the United States of America 76, 6147-6151.

Little, J. W., Edmiston, S. H., Pacelli, L. R. \& Mount, D. W. (1980). Cleavage of the Escherichia coli lexA protein by the recA protease. Proceedings of the National Academy of Sciences of the United States of America 77, 3225-3229.

LLOYD, R. G. \& BARBOUR, S. D. (1974). The genetic location of the $s b c A$ gene of Escherichia coli. Molecular and General Genetics 134, 157-171.

LLOYD, R. G. \& Low, B. (1976). Some genetic consequences of changes in the level of rec $A$ gene function in Escherichia coli K-12. Genetics 84, 675695.

McEntee, K. \& Weinstock, G. M. (1981), tif-I mutation alters polynucleotide recognition by the recA protein of Escherichia coli. Proceedings of the National Academy of Sciences of the United States of America 78, 6061-6065.

Mount, D. W., Walker, A. C. \& Kosel, C. (1973). Suppression of lex mutations affecting deoxyribonucleic acid repair in Escherichia coli $\mathrm{K}-12$ closely linked thermosensitive mutations. Journal of Bacteriology 116, 950-956.

Mount, D. W., Little, J. W. \& Edmiston, S. H. (1980). Influence of plasmids carrying the lexA gene on DNA repair and related processes in Escherichia coli K-12. Molecular and General Genetics 117, 477483.

PhizICKY, E. M. \& RoBerTs, J. W. (1981). Induction of SOS functions: regulation of proteolytic activity of $E$. coli RecA protein by interaction with DNA and nucleoside triphosphate. Cell 25, 259-267.

RADDING, C. M. (1981). Recombination activities of $E$. coli RecA protein. Cell 25, 3-4.

Ream, L. W., Margossian, L., Clark, A. J., Hansen, F. G. \& Meyenburg, K. (1980). Genetic and physical mapping of recF in Escherichia coli $\mathrm{K}-12$. Molecular and General Genetics 180, 115-121.

Rothman, R. H. \& Clark, A. J. (1977). The dependence of postreplication repair on $u v r B$ in a recF mutant of Escherichia coli $\mathrm{K}-12$. Molecular and General Genetics 155, 279-286.

Rothman, R. H., Margossian, L. J. \& Clark, A. J. (1979). W-reactivation of phage lambda in $r e c F$, recL, $u v r A$, and $u v r B$ mutants of $E$. coli $\mathrm{K}-12$. Molecular and General Genetics 169, 279-287.

Thomas, A. \& Lloyd, R. G. (1980). Altered regulation of the rec $A$ gene in Escherichia coli strains carrying a recA-linked suppressor of lexA. Molecular and General Genetics 179, 355-358.

WITKIN, E. M. (1976). Ultraviolet mutagenesis and inducible deoxyribonucleic acid repair in Escherichia coli. Bacteriological Reviews 40, 869-907.

ZARITSKY, A. \& PrITCHARD, R. H. (1973). Changes in cell size and shape associated with changes in the replication time of the chromosome of Escherichia coli. Journal of Bacteriology 114, 824-837. 